\title{
Cholesterol, lipid rafts, and disease
}

\author{
Kai Simons and Robert Ehehalt \\ Max Planck Institute of Molecular Cell Biology and Genetics, Dresden, Germany \\ J. Clin. Invest. 110:597-603 (2002). doi:10.1172/JCI200216390.
}

Lipid rafts are dynamic assemblies of proteins and lipids that float freely within the liquid-disordered bilayer of cellular membranes but can also cluster to form larger, ordered platforms. Rafts are receiving increasing attention as devices that regulate membrane function in eukaryotic cells. In this Perspective, we briefly summarize the structure and regulation of lipid rafts before turning to their evident medical importance. Here, we will give some examples of how rafts contribute to our understanding of the pathogenesis of different diseases. For more information on rafts, the interested reader is referred to recent reviews $(1,2)$.

\section{Composition of lipid rafts}

Lipid rafts have changed our view of membrane organization. Rafts are small platforms, composed of sphingolipids and cholesterol in the outer exoplasmic leaflet, connected to phospholipids and cholesterol in the inner cytoplasmic leaflet of the lipid bilayer. These assemblies are fluid but more ordered and tightly packed than the surrounding bilayer. The difference in packing is due to the saturation of the hydrocarbon chains in raft sphingolipids and phospholipids as compared with the unsaturated state of fatty acids of phospholipids in the liquid-disordered phase (3). Thus, the presence of liquid-ordered microdomains in cells transforms the classical membrane fluid mosaic model of Singer and Nicholson into a more complex system, where proteins and lipid rafts diffuse laterally within a two-dimensional liquid.

Membrane proteins are assigned to three categories: those that are mainly found in the rafts, those that are present in the liquid-disordered phase, and those that represent an intermediate state, moving in and out of rafts. Constitutive raft residents include glycophos-

\footnotetext{
Address correspondence to: Kai Simons, Max Planck Institute of Molecular Cell Biology and Genetics, Pfotenhauerstrasse 108, D-01307 Dresden, Germany. Phone: 49-351-2102800; Fax: 49-351-2102900; E-mail: simons@mpi-cbg.de. Robert Ehehalt's present address is: University of Heidelberg, Department of Internal Medicine IV, Heidelberg, Germany. Conflict of interest: No conflict of interest has been declared. Nonstandard abbreviations used: glycophosphatidylinositolanchored (GPI-anchored); endothelial nitric oxide synthase (eNOS); $\beta$-secretase (BACE); endoplasmic reticulum (ER); baby hamster kidney (BHK); detergent-resistant membranes (DRMs); Alzheimer disease (AD); amyloid- $\beta$-peptide (A $\beta)$.
}

phatidylinositol-anchored (GPI-anchored) proteins; doubly acylated proteins, such as tyrosine kinases of the Src family, $\mathrm{G} \alpha$ subunits of heterotrimeric $\mathrm{G}$ proteins, and endothelial nitric oxide synthase (eNOS); cholesterol-linked and palmitate-anchored proteins like Hedgehog (see Jeong and McMahon, this Perspective series, ref. 4); and transmembrane proteins, particularly palmitoylated proteins such as influenza virus hemagglutinin and $\beta$-secretase (BACE) (1). Some membrane proteins are regulated raft residents and have a weak affinity for rafts in the unliganded state. After binding to a ligand, they undergo a conformational change and/or become oligomerized. When proteins oligomerize, they increase their raft affinity (5). A peripheral membrane protein, such as a nonreceptor tyrosine kinase, can be reversibly palmitoylated and can lose its raft association after depalmitoylation (6). By these means, the partitioning of proteins in and out of rafts can be tightly regulated.

\section{Cholesterol and raft biogenesis}

Cholesterol is thought to serve as a spacer between the hydrocarbon chains of the sphingolipids and to function as a dynamic glue that keeps the raft assembly together (1). Cholesterol partitions between the raft and the nonraft phase, having higher affinity to raft sphingolipids than to unsaturated phospholipids. Removal of raft cholesterol leads to dissociation of most proteins from rafts and renders them nonfunctional.

Association with detergent-resistant membranes (DRMs) is a useful criterion to estimate whether a protein associates with lipid rafts (2). After solubilization of membranes or cells with Triton X-100 or with CHAPS at $4^{\circ} \mathrm{C}$, raft-associated lipids and proteins remain insoluble and can then be floated to low density by sucrose gradient centrifugation. If cholesterol is extracted by methyl- $\beta$-cyclodextrin or complexed by saponin, the raft proteins usually, but not always, become detergent-soluble.

Lipid rafts are first assembled in the Golgi complex in mammalian cells (3). Cholesterol is synthesized in the endoplasmic reticulum (ER), as is ceramide, the hydrophobic backbone of sphingolipids. However, most of the sphingolipid head groups are attached to ceramide in the Golgi complex, where raft assembly takes place (7). There is an increasing concentration of cholesterol and sphingolipids from the ER to the plas- 
Table 1

Diseases for which rafts and raft proteins are targets

Alzheimer disease

Parkinson disease

Muscular dystrophy

Polyneuropathies, demyelinating diseases

Autoimmune disease, chronic inflammation, vaccine response

$B$ cell response

$\mathrm{T}$ cell response

Asthma and allergic response

Neoplasia

Atherosclerosis

Hypertension, hemodynamic regulation

Diabetes

Hyperparathyroidism

Osteoarthritis

Gastrointestinal ulceration

Paroxysmal nocturnal hemoglobinuria

Lysosomal storage disease

Niemann-Pick disease

Tay-Sachs disease, morbus Fabry, metachromatic leukodystrophy

Pilzaeus-Merzbacher disease

Postsqualene cholesterol biosynthesis disorders

Pore-forming toxins (gas gangrene)

Sepsis, septic shock

Bacterial infections

Escherichia coli

Mycobacteria tuberculosis and bovis

Campylobacter jejuni

Vibrio cholerae

Clostridium difficile (pseudomembranous colitis)

Clostridium tetani

Salmonella, Shigella

Viral infections

Influenza virus

HIV-1

Measles virus

Respiratory syncytial cell virus

Filoviridae (Ebolavirus, Marburgvirus)

Papillomaviridae and polyomaviridae

Epstein-Barr virus

Echovirus 1

Other pathogens

Plasmodium (malaria)

Trypanosoma (sleeping sickness)

Leishmania

Prions (Creutzfeldt-Jakob disease, Kuru,

Gerstmann-Sträussler-Scheinker syndrome)

Toxoplasma gondii

See supplemental reading list (www.jci.org/cgi/content/full/110/5/597/DC1) for detailed references.

ma membrane. This increase seems to be achieved by excluding lipid rafts from the retrograde traffic between the Golgi complex and the ER (8). Thus, lipid rafts are moved forward from the Golgi complex to the plasma membrane, where they concentrate but also spread into the endocytic recycling pathways (9). Cholesterol and sphingolipid concentrations are tightly regulated and limit the supply of lipid rafts to organelles supplied by the Golgi apparatus.
The cell pays a price for using cholesterol as a spacer for keeping rafts together. Cholesterol is toxic, and its cellular levels are kept in tight control by an intricate network of transcriptional regulation of cholesterol biosynthesis and cellular uptake as well as by deposition of cholesterol into fat droplets in an esterified form, and by cellular efflux (ref. 10; see also Tall et al. [ref. 11] and Tabas [ref. 12], this Perspective series). Disturbance of these tightly regulated processes leads to a variety of diseases of lipid metabolism, as shown in Table 1.

\section{Regulation of raft size}

One important issue in raft function is their size. There is consensus that rafts are too small to be resolved by light microscopy. Pralle et al. (13) employed photonic force microscopy to measure the size of lipid rafts and found that rafts in the plasma membrane of fibroblasts diffuse as assemblies of $50 \mathrm{~nm}$ diameter, corresponding to a surface area covered by about 3,000 sphingolipids. Based on data from cultured baby hamster kidney (BHK) cells, whose lipid composition and organelle surface area have been examined in detail, it appears that an individual cell has a surface area of approximately $2,000 \mu \mathrm{m}^{2}$. The lipid composition of the cell plasma membrane contains $26 \%$ phosphatidylcholine, $24 \%$ sphingomyelin, and $12 \%$ glycosphingolipids. Due to the asymmetric nature of the lipid organization in the plasma membrane, most of the sphingolipids occupy the outer leaflet of the bilayer, while less than half of the phosphatidylcholine has been estimated to be in this leaflet (14).

Assuming that most of the sphingolipid is raft-associated, rafts would cover more than half of the cell surface. The density of membrane proteins has been estimated to be around 20,000 molecules per $\mu \mathrm{m}^{2}$. Thus, the plasma membrane would accordingly contain about $40 \times 10^{6}$ protein molecules. The number of 50$\mathrm{nm}$ rafts would be about $10^{6}$, and if the density of proteins is the same in rafts as in the surrounding bilayer, each raft would carry about 20 protein molecules. If BHK cells are representative, it follows that the density of rafts floating in the fibroblast plasma membrane is high. If $20 \times 10^{6}$ raft protein molecules were distributed more or less randomly, each raft would likely contain a different subset of proteins. A kinase attached to the cytosolic leaflet of a raft is, therefore, unlikely to meet its substrate in the same individual raft.

The small size of an individual raft may be important for keeping raft-borne signaling proteins in the "off" state. Accordingly, for activation to occur, many rafts have to cluster together, forming a larger platform, where the protein participants in a signal transduction process can meet, undisturbed by what happens outside the platform (Figure 1). Thus, rafts are small, and, when activated, they cluster to form larger platforms in which functionally related proteins can interact. One way to analyze raft association and clustering is to patch raft and nonraft components on the surface of living cells by specific antibodies $(5,15)$. If two raft components are cross-linked by antibodies, they will form overlapping patches in the plasma 
membrane. However, patching of a raft protein and a nonraft marker such as the transferrin receptor leads to the formation of segregated patches. In general, copatching of two raft components is dependent on the simultaneous addition of both antibodies to the cells. If antibodies are added sequentially, segregated patches predominate. Notably, the patching behavior is cholesterol-dependent. As a consequence of the small size and the heterogeneous composition of individual rafts, these structures must be clustered in specific ways if signaling is to ensue.

One example of such a raft clustering process encountered in daily clinical practice is the $\mathrm{IgE}$ signaling during the allergic immune response $(16,17)$. The allergen that elicits the allergic reaction by stimulating the degranulation of a mast or basophilic cell is multivalent, binding several IgE antibody molecules. Cross-linking of two or more $\operatorname{IgE}$ receptors $[\mathrm{Fc}(\varepsilon) \mathrm{RI}]$ increases their association with rafts, as measured by increased detergent resistance. Within the rafts, cross-linked $\mathrm{Fc}(\varepsilon) \mathrm{RI}$ becomes tyrosinephosphorylated by raft-associated Lyn, a double-acylated Src-related kinase. The $\mathrm{Fc}(\varepsilon) \mathrm{RI}$ phosphorylation recruits Syk-related kinases, which are activated and lead to binding and scaffolding of downstream signaling molecules and, finally, to the formation of a signaling platform. This structure includes the raft protein LAT (linker of activation of T cells), which guides the clustering of additional rafts into the expanding platform (18). Signaling leads to calcium mobilization, which triggers the release of preformed mediators such as histamine from the intracellular stores.

The more participants are collected into the raft platform, the higher the signaling response. Uncontrolled amplification of the signaling cascade by raft clustering might trigger hyperactivation, with life-threatening consequences such as Quinke edema and allergic shock. The whole signaling assembly can be dissociated by dephos- phorylation or downregulated by internalization of the components by endocytosis (19). Thus, in IgE signaling, lipid rafts serve to increase the efficiency by concentrating the participating proteins into fluid microdomains and limiting their lateral diffusion so that proteins remain at the site of signaling. Even a small change of partitioning into lipid rafts can, through amplification, initiate a signaling cascade or prompt a deleterious overshoot, as occurs in allergic reactions (20).

Another clinically relevant example of raft clustering is the pathogenic mechanisms of pore-forming toxins, which are secreted by Clostridium, Streptococcus, and Aeromonas species, among other bacteria (21). These toxins may cause diseases ranging from mild cellulitis to gaseous gangrene and pseudomembranous colitis. Best studied is the toxin aerolysin from the marine bacterium Aeromonas bydrophila (22). Aerolysin is secreted and binds to a GPI-anchored raft protein on the surface of the host cell. The toxin is incorporated into the membrane after proteolysis and then heptamerizes in a raft-dependent manner to form a raft-associated channel through which small molecules and ions flow to trigger the pathogenic changes. The oligomerization of aerolysin can be triggered in solution but occurs at more than $10^{5}$-fold lower toxin concentration at the surface of the living cell (22). This enormous increase in efficiency is due to activation by raft binding and by concentration into raft clusters, which is driven by the oligomerization of the toxin. Again, a small change can lead to a huge effect by amplification of raft clustering.

\section{Caveolae and lipid rafts}

One source of confusion in the raft field has been the interrelationship between caveolae and rafts. Fortunately, this issue has now been cleared up by the analysis of mice deficient in caveolins (23-25). Caveolae are small surface invaginations seen in many cell types (26). Some
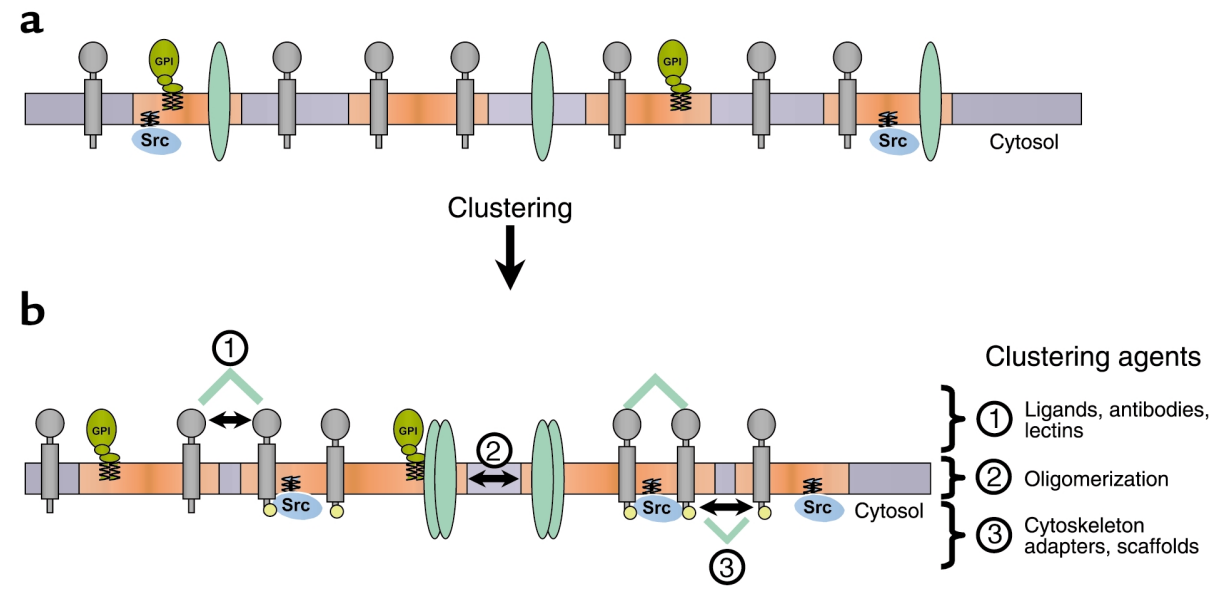

\footnotetext{
Figure 1

Mechanisms of raft clustering. (a) Rafts (red) are small at the plasma membrane, containing only a subset of proteins. (b) Raft size is increased by clustering, leading to a new mixture of molecules. This clustering can be triggered (1) at the extracellular side by ligands, antibodies, or lectins, (2) within the membrane by oligomerization, or (3) by cytosolic agents (cytoskeletal elements, adapters, scaffolds). Raft clustering occurs at the plasma membrane as well as intracellularly, e.g., in endosomal lumen. Ligand binding or oligomerization can alter the partitioning of proteins in and out of rafts. Increased raft affinity of a given protein and its activation within rafts (e.g., phosphorylation by Src-family kinases [yellow]) can initiate a cascade of events, leading to further increase of raft size by clustering.
} 
cells, such as adipocytes, endothelial cells, and smooth muscle cells, have plasma membranes with numerous caveolae, which have been postulated to be formed by clustering of rafts on the cell surface.

Caveolar invagination is driven by polymerization of caveolins, of which there are three: caveolin-1, -2 , and 3 . All caveolins are raft-associated, and mice deficient in caveolins lack caveolae (24). The mice are superficially healthy at birth, but detailed analysis shows that they have exercise intolerance accompanied by severe lung abnormalities, endothelial cell proliferations, and fibrosis, as well as dysregulation of the vascular tone; this last effect is secondary to eNOS activation and disturbance of calcium signaling. Caveolin-1-deficient animals also develop a lung pathology resembling the end-state of a range of parenchymal lung disorders, including industrial lung diseases, sarcoidosis and connective tissue diseases, and idiopathic pulmonary fibrosis and chronic hypersensitivity pneumonitis (reviewed in ref. 27). Mutations in the human gene for the muscle-specific caveolin-3 lead to an autosomal dominant form of limb-girdle muscular dystrophy. In this disease, there is severe impairment of caveola formation in the striated muscle fibers, combined with a disorganization of the T-tubule system (28). The analysis of the caveolin-deficient mice has shown that most lipid raft functions are normal. Caveolae thus appear to have relatively narrow, tissue-specific functions.

Like rafts, caveolae are dynamic structures. Caveolae usually remain attached to the cell surface, but they can be endocytosed, for instance when they encounter Simian virus-40 (29). The ligand-triggered endocytosis of the virus is regulated by phosphorylation. After binding of the virus, it takes 20 minutes or more before caveolae pinch off from the plasma membrane and move into the cell. Video microscopy demonstrates that caveolae devoid of virus particles do not internalize. Cross-linking of raft proteins and lipids on the cell surface may result in movement into caveolae, where the cross-linked complexes become trapped. One physiological example is the inactivation of eNOS; after activation, this enzyme moves into caveolae, where the enzyme is downregulated (30). Normally, caveolae may primarily serve to store and downregulate raft proteins or act as reservoirs of rafts. Why some cells, such as adipocytes, have plasma membranes almost covered by caveolae remains an enigma.

Lipid rafts play a central role in many cellular processes, including membrane sorting and trafficking, cell polarization, and signal transduction processes that have been best studied in T cells (15), B cells (31), and, as mentioned, the allergic response $(16,17)$. Ceramide/sphingomyelin signaling, which regulates cell growth, survival, and death, also involves raft clustering (32). Several groups of pathogens, bacteria, prions, viruses, and parasites hijack lipid rafts for their purposes (33). The already impressive catalog of raft involvement in disease (Table 1) continues to grow. Below, we illustrate the principles that are emerging from studies on raft functions in physiology and human disease by focusing on viruses, prions, and Alzheimer disease.

\section{Viral infection}

Several viruses employ lipid rafts to infect host cells (Table 1). The first example to be characterized was influenza virus (34). The virus contains two integral glycoproteins, hemagglutinin and neuraminidase, both of which are raft-associated as judged by cholesterol-dependent detergent resistance (35). Influenza virus buds out from the apical membrane of epithelial cells, which is enriched in raft lipids. Influenza virus preferentially includes raft lipids in its envelope during budding, a process in which polymerization of $\mathrm{M}$ proteins forms a layer facing the cytosolic side of the nascent viral envelope and thus drives raft clustering (35). HIV-1, which likewise incorporates host raft lipids and proteins into its envelope, employs rafts for at least four key events in its life cycle: passage across a new host's mucosa, viral entry into immune cells, signaling of changes in host cell functions as well as viral exit from cells, and dispersion through the host's vascular system.

In the first process, breaching a host animal's mucosa, HIV binds to the glycosphingolipid galactosylceramide at the apical surface of mucosal epithelial cells and then transcytoses across the epithelium to be released on the basolateral side. Disrupting raft association blocks viral transcytosis (36).

During infection of target cells, the viral envelope components, as well as the internal Gag protein (which is essential for assembly of the viral envelope; ref. 37), are all initially associated with rafts defined operationally as DRMs. Indeed, viral glycoproteins can co-patch with known raft-associated proteins on the surface of living cells after cross-linking with specific antibodies (38). Interestingly, the virus receptors on the host cell surface are also raftassociated. Manes et al. (38) found that the HIV glycoprotein gp120 co-patches with the cell surface receptor $\mathrm{CD} 4$ and with the co-receptors, the chemokine receptors CCR5 and CXLR4. CD4, CCR5, and CXLR4 are found in DRMs. Binding of the virus to its surface receptors, first to CD4 and then to the chemokine receptor, seems to lead to raft clustering and lateral assembly of a protein complex in the membrane to initiate fusion of the virus envelope with the cell membrane. Both cholesterol and specific glycosphingolipid species serve as crucial elements in organizing the fusion complex $(39,40)$.

An increasing body of data shows that viruses, bacteria, and parasites prepare their way into the host cell by changing the cellular state of signaling. This is also the case during HIV infection. Nef, an early HIV gene product, promotes infectivity of the virus via lipid rafts (41); infection with HIV-1 virions lacking Nef does not progress to AIDS (42). The Nef protein is a peripheral, myristoylated membrane protein with a proline-rich repeat that can bind to raft-associated nonreceptor tyrosine kinases of the Src family. It associates with DRMs and seems to prime the host cells for HIV infection by lowering the threshold necessary for $\mathrm{T}$ cell activation (41). Resting $\mathrm{T}$ cells do not support a productive HIV infection, but Nef activates $T$ 
cells by increasing IL-2 secretion and obviates the need for costimulatory signals. By clustering lipid rafts carrying relevant host cell surface proteins, Nef oligomerization may aid in organizing the $\mathrm{T}$ cell signaling complex and the HIV budding site $(41,43)$.

HIV exit from the cell, another raft-dependent step, depends critically on the viral Gag protein $(39,44)$. Viruses contain 1,200-1,500 Gag molecules, which multimerize on the cytosolic leaflet of the membrane, driving viral assembly and budding. In this process the Gag-Gag interactions collect the virus spike proteins to the bud site. This process requires palmitoylation of gp120 and myristoylation of Gag, and it can be blocked by cholesterol depletion (39). Thus, one can envisage that Gag proteins specifically bind to rafts containing HIV spike proteins, which cluster rafts together to promote virus assembly. The interaction between HIV-1 protein and lipid rafts may cause a conformational change in Gag required for envelope assembly (37).

\section{Alzheimer disease}

Alzheimer disease (AD) is becoming an interesting example of how lipid rafts are involved in regulating protein trafficking and processing. Formation of senile plaques containing the amyloid- $\beta$-peptide $(A \beta)$ is a hallmark of $\mathrm{AD}$. $\mathrm{A} \beta$, a fragment derived from the large type I transmembrane protein APP, the amyloid precursor protein (45), is cleaved sequentially by enzymes termed $\beta$-secretase (BACE) and $\gamma$-secretase.

BACE is a novel aspartyl-protease that sets the pace of $A \beta$ generation. BACE cleaves APP in its luminal domain, generating a secreted ectodomain. The resulting $10-\mathrm{kDa} \mathrm{C}$-terminal fragment is subsequently clipped by $\gamma$-secretase, which acts at the transmembrane domain of APP to release A $\beta$. A third enzymatic activity, the $\alpha$-secretase, cleaves APP in the middle of the $A \beta$ region, generating the $\alpha$ fragment (a secreted ectodomain), as well as a short C-terminal stub that is also cleaved by $\gamma$-secretase. Cleavage of $\alpha$ cuts APP within the $\mathrm{A} \beta$ region, yielding products that are non-amyloidogenic. Importantly, $\alpha$-cleavage directly competes with $\beta$-cleavage for their common substrate APP. Therefore, a key issue for understanding how the disease-promoting peptide $A \beta$ is generated is the question of how access of these enzymes to APP is regulated.

There is growing evidence that cholesterol plays a central role in regulating $\alpha$ - and $\beta$-cleavage (46). First, the $\varepsilon 4$ allele of apoE, a carrier that plays a major role in cholesterol delivery within the CNS, represents a major risk factor for AD. Levels of total cholesterol and LDL in serum apparently correlate with the amount of $A \beta$ in $A D$ brains, and there is epidemiological evidence that elevated cholesterol level during mid-life increases the risk of developing AD (47). In addition, elevated dietary cholesterol uptake also increases amyloid plaque formation in transgenic mice and rabbits. Recently, two independent retrospective studies reported a strong decrease in the incidence of $\mathrm{AD}$ and dementia in patients treated with 3-hydroxy3-methylglutaryl-CoA-reductive (HMG-CoA-reductase) inhibitors $(48,49)$.
Most importantly, it has been shown that cholesterol depletion inhibits $\beta$-cleavage and $A \beta$ formation in neurons and other cells, while at the same time promoting $\alpha$-cleavage (46). We hypothesize that APP is present in two cellular pools, one associated with lipid rafts, in which $A \beta$ is generated, and another outside of rafts, where $\alpha$-cleavage takes place. This model would explain how the same protein can be processed in mutually exclusive ways. Cholesterol depletion would shift the partitioning of APP from lipid rafts to the surrounding lipid bilayer, and the extent of this shift would determine the magnitude of BACE processing. Because cell surface rafts are small and contain only a subset of proteins, the likelihood that APP and BACE are in the same rafts is low. Therefore, $\beta$-cleavage is predicted to be limited on the cell surface, although it may occur after endocytosis, when internalized raft membrane can cluster and become redistributed.

Recent evidence from our laboratory shows that APP and BACE co-patch with one another following antibody cross-linking, to form a complex that also contains GPIanchored raft markers but excludes the non-raft-associated transferrin receptor. A fraction of APP and BACE is found in DRMs, especially following antibody cross-linking. Remarkably, $A \beta$ generation is strongly reduced after expression of a mutant form of dynamin or after activation of the Rab5 GTPase-activating protein, both of which treatments inhibit endocytosis. Conversely, $A \beta$ production is strongly stimulated after cross-linking, presumably because clustering brings together surface rafts containing APP and BACE. As would be predicted, the effect of clustering is not sensitive to blockade by inhibition of endocytosis (R. Ehehalt et al., unpublished data).

Even after processing, interactions between $A \beta$ and rafts may affect Alzheimer pathogenesis. Thus, raftderived $A \beta$ has been shown to promote fibrillogenesis of soluble $A \beta$, leading Mizuno et al. (50) to suggest that raft binding causes a conformational change that promotes amyloid plaque formation. Cholesterol depletion reduces the seeding properties of $A \beta$. Another raft component, the ganglioside GM1, is known to bind $A \beta$ and perhaps change the latter's conformation (51). All these studies suggest a critical and decisive role for lipid rafts in $A \beta$ generation.

\section{Prion diseases}

A conformational change resulting in amyloid formation is also involved in the pathogenesis of prion disease. Prion diseases are thought be promoted by an abnormal form (PrPsc) of a host-encoded protein $(\mathrm{PrPc})$. PrPsc can interact with its normal counterpart $\mathrm{PrPc}$ and change the conformation of PrPc so that the protein turns into PrPsc. PrPsc then self-aggregates in the brain, and these aggregates are thought to cause the disorders manifested in humans as Creutzfeldt-Jakob disease, Kuru, or Gerstmann-Sträussler-Scheinker syndrome (52). The mechanism by which PrPc is converted to PrPsc is not known, but several lines of evidence suggest that lipid rafts are involved $(53,54)$.

PrP is a GPI-anchored protein. Both PrPc and PrPsc are associated with DRMs in a cholesterol-dependent 
manner. Cholesterol depletion of cells leads to decreased formation of PrPsc from PrPc. The GPI anchor is required for conversion. When the GPI anchor is exchanged with a transmembrane domain, conversion to abnormal proteins is blocked. In vitro, the conversion of $\mathrm{PrPc}$ to $\mathrm{PrPsc}$, as monitored by $\mathrm{PrP}$ protease resistance, occurs when microsomes containing PrPsc are fused with DRMs containing PrP (53). Extraction with detergent leads to raft clustering in DRMs. Fusion of microsomes with DRMs was necessary in this experiment because simply mixing the membranes did not lead to measurable generation of new PrPsc. On the other hand, releasing PrP ectodomains from PrPsc by phospholipase $\mathrm{C}$ treatment also stimulated conversion of PrP to PrPsc in this system. Baron et al. (53) hypothesize that membrane components exchange between apposed cells; a possible mechanism for such an exchange is that the cells release membrane vesicles containing PrPsc that fuse with neighboring cells. Indeed, a similar process has been found to mediate transfer of the raft-associated chemokine receptor CCR5 (55). Alternatively, GPI-anchored PrPsc could be released as such from one cell and move across the extracellular aqueous phase to be inserted into another cell. Recently, it was shown that direct cell-cell contact is required for transfer of PrPsc infectivity in cell culture (56).

How lipid rafts promote abnormal prion conversion is not clear. No in vitro generated PrPsc has yet been shown to be infectious. Again, however, because of the small size of individual rafts, plasma membrane PrPc and PrPsc would in all likelihood be in separate rafts and therefore would not meet each other. Endocytosis has also been shown to play a role for prion conversion, as is the case for BACE cleavage of APP. We hypothesize that rafts containing PrPc and PrPsc become clustered after endocytosis. It is also possible that the protein factor $\mathrm{X}$, postulated to mediate conversion, is involved in raft clustering after endocytosis. In support of this scenario is the finding that a $\mathrm{pH}$ of $6-7$, which prevails in endosomes, promotes conversion. If $\mathrm{PrPc}$ and PrPsc were clustered into the same raft platform after endocytosis, an increase of interaction efficiency would result and lead to amplification of conversion.

Obviously more work is required to sort out this remarkable process, but since raft lipids have proved to be required for prion conversion to proceed efficiently, it may be that these structures serve not only to concentrate the components but also to directly regulate the conformational changes that yield infectious prions.

\section{Perspectives}

Research on lipid rafts is now entering an exciting phase. From what is already known, rafts represent versatile devices for compartmentalizing cellular membrane processes. In the nonactivated state they float freely, carrying a few passenger proteins, but, when activated, they coalesce to form larger platforms where proteins meet to perform functions in signaling, processing, and transport. Clustering of rafts is key to understanding raft function in human physiology as well as in development of human disease.
The biophysics of raft assembly is still poorly understood. How is the inner leaflet composed and linked to outer leaflet of raft assemblies? What limits the size of rafts in nonactivated and activated states? What role do the different constituents play? There is evidence for specific functions for different glycosphingolipids and phosphoinositides in rafts, but little is known about how these lipids provide specificity to membrane function in linking to proteins. What determines raft association of proteins and lipids? How is raft scaffolding accomplished? What is the role of the actin cytoskeleton, and how is the concentration of raft lipids in membranes regulated? A great deal is known about cholesterol regulation, but how are the different sphingolipid levels adjusted? Novel mass spectroscopic methodology is already available to analyze both proteins and lipids in membranes, but the field will still require additional tools to elucidate how lipids and proteins interact in cell membranes.

\section{Acknowledgments}

We thank T. Kurzchalia, W. Stremmel, and the members of the Simons laboratory for critical reading of this manuscript. The authors were supported by the Deutsche Forschungsgemeinschaft Schwerpunktprogramm SPP 1085, Zelluläre Mechanismen der Alzheimer Erkrankung.

1. Simons, K., and Toomre, D. 2000. Lipid rafts and signal transduction. Nat. Rev. Mol. Cell Biol. 1:31-39.

2. London, E., and Brown, D.A. 2000. Insolubility of lipids in triton X-100: physical origin and relationship to sphingolipid/cholesterol membrane domains (rafts). Biochim. Biophys. Acta. 1508:182-195.

3. Brown, D.A., and London, E. 1998. Functions of lipid rafts in biological membranes. Annu. Rev. Cell Dev. Biol. 14:111-136.

4. Jeong, J., and McMahon, A.P. 2002. Cholesterol modification of Hedgehog family proteins. J. Clin. Invest. 110:591-596. doi:10.1172/JCI200216506.

5. Harder, T., et al. 1998. Lipid domain structure of the plasma membrane revealed by patching of membrane components. J. Cell Biol. 141:929-942.

6. Zacharias, D.A., et al. 2002. Partitioning of lipid-modified monomeric GFPs into membrane microdomains of live cells. Science. 296:913-916.

7. van Meer, G. 1989. Lipid traffic in animal cells. Annu. Rev. Cell Biol. 5:247-275.

8. Brugger, B., et al. 2000. Evidence for segregation of sphingomyelin and cholesterol during formation of COPI-coated vesicles. J. Cell Biol. 151:507-518.

9. Mukherjee, S., and Maxfield, F.R. 2000. Role of membrane organization and membrane domains in endocytic lipid trafficking. Traffic. 1:203-211.

10. Simons, K., and Ikonen, E. 2000. How cells handle cholesterol. Science. 290:1721-1726.

11. Tall, A.R., Costet, P., and Wang, N. 2002. Regulation and mechanisms of macrophage cholesterol efflux. J. Clin. Invest. In press.

12. Tabas, I. 2002. Consequences of cellular cholesterol accumulation: basic concepts and physiological implications. J. Clin. Invest. In press.

13. Pralle, A., et al. 2000. Sphingolipid-cholesterol rafts diffuse as small entities in the plasma membrane of mammalian cells. J. Cell Biol. 148:997-1008.

14. Allan, D. 1996. Mapping the lipid distribution in the membranes of BHK cells (mini-review). Mol. Membr. Biol. 13:81-84.

15. Janes, P.W., et al. 2000. The role of lipid rafts in $\mathrm{T}$ cell antigen receptor (TCR) signaling. Semin. Immunol. 12:23-34.

16. Sheets, E.D., Holowka, D., and Baird, B. 1999. Membrane organization in immunoglobulin E receptor signaling. Curr. Opin. Chem. Biol. 3:95-99.

17. Holowka, D., and Baird, B. 2001. Fc(epsilon)RI as a paradigm for a lipid raftdependent receptor in hematopoietic cells. Semin. Immunol. 13:99-105.

18. Rivera, J., et al. 2001. A perspective: regulation of IgE receptor-mediated mast cell responses by a LAT-organized plasma membrane-localized signaling complex. Int. Arch. Allergy Immunol. 124:137-141.

19. Xu, K., et al. 1998. Stimulated release of fluorescently labeled IgE fragments that efficiently accumulate in secretory granules after endocytosis in RBL2H3 mast cells. J. Cell Sci. 111:2385-2396.

20. Kholodenko, B.N., Hoek, J.B., and Westerhoff, H.V. 2000. Why cytoplasmic signaling proteins should be recruited to cell membranes. Trends Cell Biol. 10:173-178.

21. Lesieur, C., et al. 1997. Membrane insertion: the strategies of toxins (review). Mol. Membr. Biol. 14:45-64.

22. Abrami, L., and van Der Goot, F.G. 1999. Plasma membrane microdomains 
act as concentration platforms to facilitate intoxication by aerolysin. J. Cell Biol. 147:175-184.

23. Razani, B., et al. 2002. Caveolin-2-deficient mice show evidence of severe pulmonary dysfunction without disruption of caveolae. Mol. Cell. Biol. 22:2329-2344.

24. Drab, M., et al. 2001. Loss of caveolae, vascular dysfunction, and pulmonary defects in caveolin-1 gene-disrupted mice. Science. 293:2449-2452.

25. Galbiati, F., et al. 2001. Caveolin-3 null mice show a loss of caveolae, changes in the microdomain distribution of the dystrophin-glycoprotein complex, and t-tubule abnormalities. J. Biol. Chem. 276:21425-21433.

26. Kurzchalia, T.V., and Parton, R.G. 1999. Membrane microdomains and caveolae. Curr. Opin. Cell Biol. 11:424-431.

27. Razani, B., and Lisanti, M.P. 2001. Caveolin-deficient mice: insights into caveolar function and human disease. J. Clin. Invest. 108:1553-1561. doi:10.1172/JCI200114611.

28. Minetti, C., et al. 2002. Impairment of caveolae formation and T-system disorganization in human muscular dystrophy with caveolin-3 deficiency. Am. J. Pathol. 160:265-270.

29. Pelkmans, L., and Helenius, A. 2002. Endocytosis via caveolae. Traffic. 3:311-320.

30. Sowa, G., Pypaert, M., and Sessa, W.C. 2001. Distinction between signaling mechanisms in lipid rafts vs. caveolae. Proc. Natl. Acad. Sci. USA. 98:14072-14077.

31. Cherukuri, A., Dykstra, M., and Pierce, S.K. 2001. Floating the raft hypothesis: lipid rafts play a role in immune cell activation. Immunity. 14:657-660.

32. Kolesnick, R. 2002. The therapeutic potential of modulating the ceramide/sphingomyelin pathway. J. Clin. Invest. 110:3-8. doi:10.1172/JCI200216127.

33. van der Goot, F.G., and Harder, T. 2001. Raft membrane domains: from a liquid-ordered membrane phase to a site of pathogen attack. Semin. Immunol. 13:89-97.

34. Scheiffele, P., et al. 1999. Influenza viruses select ordered lipid domains during budding from the plasma membrane. J. Biol. Chem. 274:2038-2044.

35. Zhang, J., Pekosz, A., and Lamb, R.A. 2000. Influenza virus assembly and lipid raft microdomains: a role for the cytoplasmic tails of the spike glycoproteins. J. Virol. 74:4634-4644.

36. Alfsen, A., et al. 2001. Secretory IgA specific for a conserved epitope on gp41 envelope glycoprotein inhibits epithelial transcytosis of HIV-1. J. Immunol. 166:6257-6265.

37. Campbell, S.M., Crowe, S.M., and Mak, J. 2001. Lipid rafts and HIV-1: from viral entry to assembly of progeny virions. J. Clin. Virol. 22:217-227.

38. Manes, S., et al. 2000. Membrane raft microdomains mediate lateral assemblies required for HIV-1 infection. EMBO Rep. 1:190-196.

39. Ono, A., and Freed, E.O. 2001. Plasma membrane rafts play a critical role in HIV-1 assembly and release. Proc. Natl. Acad. Sci. USA. 98:13925-13930.

40. Hug, P., et al. 2000. Glycosphingolipids promote entry of a broad range of human immunodeficiency virus type 1 isolates into cell lines expressing CD4, CXCR4, and/or CCR5. J. Virol. 74:6377-6385.

41. Zheng, Y.H., et al. 2001. Nef increases infectivity of HIV via lipid rafts. Curr. Biol. 11:875-879.

42. Kirchhoff, F., et al. 1995. Brief report: absence of intact nef sequences in a long-term survivor with nonprogressive HIV-1 infection. N. Engl. J. Med. 332:228-232.

43. Wang, J.K., et al. 2000. The Nef protein of HIV-1 associates with rafts and primes T cells for activation. Proc. Natl. Acad. Sci. USA. 97:394-399.

44. Lindwasser, O.W., and Resh, M.D. 2001. Multimerization of human immunodeficiency virus type $1 \mathrm{Gag}$ promotes its localization to barges, raft-like membrane microdomains. J. Virol. 75:7913-7924.

45. Selkoe, D.J. 2001. Alzheimer's disease: genes, proteins, and therapy. Physiol. Rev. 81:741-766.

46. Simons, M., et al. 2001. Cholesterol and Alzheimer's disease: is there a link? Neurology. 57:1089-1093.

47. Kivipelto, M., et al. 2001. Midlife vascular risk factors and Alzheimer's disease in later life: longitudinal, population based study. BMJ. 322:1447-1451.

48. Jick, H., et al. 2000. Statins and the risk of dementia. Lancet. 356:1627-1631.

49. Wolozin, B., et al. 2000. Decreased prevalence of Alzheimer disease associated with 3-hydroxy-3-methyglutaryl coenzyme A reductase inhibitors. Arch. Neurol. 57:1439-1443.

50. Mizuno, T., et al. 1999. Cholesterol-dependent generation of a seeding amyloid beta-protein in cell culture. J. Biol. Chem. 274:15110-15114.

51. Choo-Smith, L.P., et al. 1997. Acceleration of amyloid fibril formation by specific binding of Abeta-(1-40) peptide to ganglioside-containing membrane vesicles. J. Biol. Chem. 272:22987-22990.

52. Caughey, B. 2000. Transmissible spongiform encephalopathies, amyloidoses and yeast prions: common threads? Nat. Med. 6:751-754.

53. Baron, G.S., et al. 2002. Conversion of raft associated prion protein to the protease-resistant state requires insertion of $\operatorname{PrP}-\mathrm{res}(\operatorname{PrP}(\mathrm{Sc}))$ into contiguous membranes. EMBO J. 21:1031-1040.

54. Taraboulos, A., et al. 1995. Cholesterol depletion and modification of $\mathrm{COOH}$-terminal targeting sequence of the prion protein inhibit formation of the scrapie isoform. J. Cell Biol. 129:121-132.

55. Mack, M., et al. 2000. Transfer of the chemokine receptor CCR5 between cells by membrane-derived microparticles: a mechanism for cellular human immunodeficiency virus 1 infection. Nat. Med. 6:769-775.

56. Kanu, N., et al. 2002. Transfer of scrapie prion infectivity by cell contact in culture. Curr. Biol. 12:523-530. 